\title{
Glycine Reduces Novelty- and Methamphetamine-Induced Locomotor Activity in Neonatal Ventral Hippocampal Damaged Rats
}

Kozo Kato, M.D., Ph.D., Toshiaki Shishido, M.D., Ph.D., Masami Ono, M.D., Kyoko Shishido, M.D., Masanori Kobayashi, M.D., and Shin-ich Niwa, M.D., Ph.D.

The use of neonatal ventral hippocampal $n V H$ lesioned rats is well established in animal models of schizophrenia.

Moreover, the dysfunction of N-methyl-D-aspartate (NMDA) neurotransmission may play a crucial role in the pathophysiology of schizophrenia. To examine the effect of glycine (GLY) in this animal model, we compared the effects of $G L Y(0.8$ and $1.6 \mathrm{~g} / \mathrm{kg}, I P)$ on locomotor activity induced by a novel environment (NOVEL) and methamphetamine $(M A P, 1.5 \mathrm{mg} / \mathrm{kg}, I P)$ in lesioned and sham-operated rats. Compared with sham rats, GLY significantly reduced
NOVEL- and MAP-induced locomotor activity in lesioned rats $(\mathrm{p}<.001$ and $\mathrm{p}<.05$, respectively). It is suggested that GLY attenuated $n V H$-induced hyperactivity, and that this effect was evident both in the presence and absence of $M A P$. The $n V H$ lesions may result in a form of hyperactivity that differs from normal locomotion in the degree to which it is highly sensitive to regulation by GLY. [Neuropsychopharmocology 24:330-332, 2001] (C) 2001 American College of Neuropsychopharmacology. Published by Elsevier Science Inc.
KEY WORDS: Schizophrenia; Hippocampus; Animal models; Glycine; Methamphetamine; Ibotenic acid

Phencyclidine (PCP) induces a psychotic state that closely resembles schizophrenia by blocking neurotransmission mediated at $N$-methyl-D (NMDA)-aspartate-type glutamate receptors, indicating that the dysfunction of endogenous NMDA receptors may play a crucial role in the pathophysiology of schizophrenia (Javitt and Zukin 1991). The NMDA receptors are stimulated not only by glutamate but also by glycine (GLY), an amino acid that acts as an obligatory co-agonist at the strychnine-

From the Department of Neuropsychiatry, Fukushima Medical University, School of Medicine, Fukushima, Japan.

Address correspondence to: Kozo Kato, M.D., Ph.D., Department of Neuropsychiatry, Fukushima Medical University, School of Medicine, 1 Hikarigaoka, Fukushima City, Fukushima 960-1295, Japan. Fax: +81 24548 6735; E-mail: kkozo@fmu.ac.jp

Received April 13, 2000; revised September 1, 2000; accepted September 12, 2000. insensitive GLY modulatory site of the NMDA receptor (Johnson and Ascher 1987; Kleckner and Dingledine 1988). It has been reported that GLY significantly reduces negative symptoms of schizophrenia (Goff and Evins 1998; Heresco-Levy et al. 1999). Additionally, GLY reduces PCP-induced but not amphetamine (a type of dopamine agonist)-induced hyperactivity in intact rats (Javitt et al. 1997).

Neonatal ventral hippocampal $(\mathrm{nVH})$ damaged rat models, based on the neurodevelopmental disorder hypothesis of schizophrenia, have recently been shown to share many similarities with schizophrenia (e.g. susceptibility to stress, dopaminergic hypersensitivity, and postpubertal onset) (Lipska et al. 1993; Lipska and Weinberger 1996). However, there have been few studies on the dysfunction of NMDA-type glutamatergic neurotransmission in this animal model. We recently reported that this animal model showed hyperresponsiveness to PCP, which does not depend on the extra- 


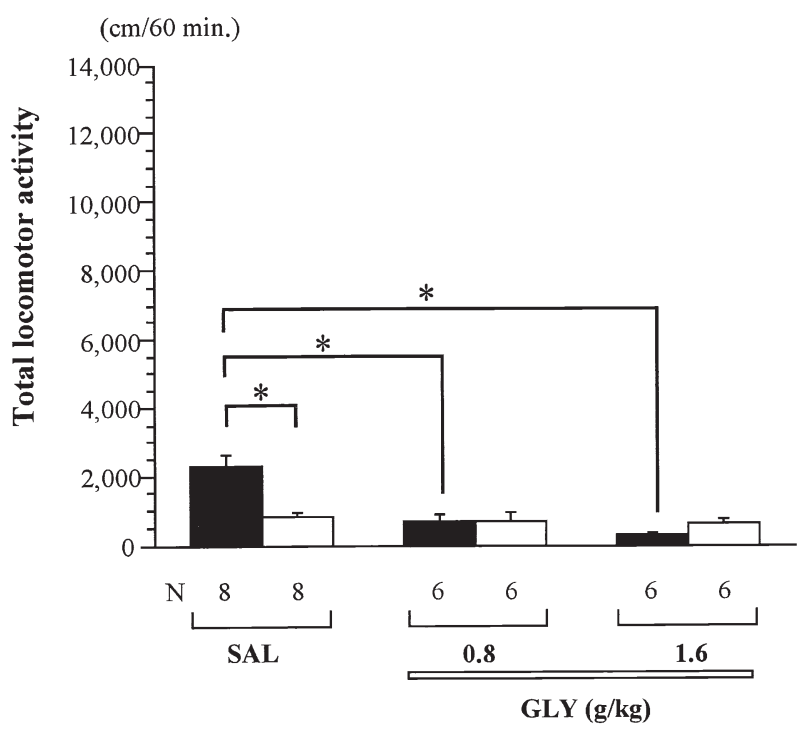

Figure 1. A novel environment (NOVEL). Locomotor activity of sham-operated (Sham, open-bar) or ibotenic acidlesioned (Lesion, closed-bar) rats tested at PD56 (means \pm SEM). Lesion and Sham rats were pretreated with either 0.8 or $1.6 \mathrm{~g} / \mathrm{kg}$, IP of glycine (GLY) or saline (SAL) $30 \mathrm{~min}$ before the behavioral testing. The total locomotor activity (ordinate) indicates summed activities during the testing period of $60 \mathrm{~min}$. Newman-Keuls post-hoc comparisons were used for comparisons between groups $\left({ }^{*} p<.001\right) . \mathrm{N}$, the number of animals.

cellular concentration of dopamine in the nucleus accumbens (Kato et al. 2000). Although those findings showed dysfunction of NMDA-type glutamatergic neurotransmission in this animal model, it is still unclear whether GLY can attenuate $\mathrm{nVH}$-induced hyperactivity.

In the present study, we examined the effects of GLY on novelty- and methamphetamine-induced locomotor activity in $\mathrm{nVH}$ lesioned rats. All in vivo procedures were carried out under the control of the Animal Research Committee in accordance with the Guidelines on Animal Experiments in Fukushima Medical University.

Rat pups were lesioned as described previously (Kato et al. 2000). Briefly, for the postnatal day 7 (PD 7) lesion, we injected $0.3 \mu \mathrm{l}$ of ibotenic acid (IBO, $10 \mu \mathrm{g} / \mu \mathrm{l})$ or an equal volume of the artificial cerebrospinal fluid into the rat pups veneral hippocampus bilaterally. Methamphetamine hydrochloride (MAP) and glycine (GLY) were dissolved in isotonic saline (SAL) before use. At PD 56, the locomotor activity of sham-operated (Sham) and IBO-lesioned (Lesion) rats was assessed in photocell activity boxes connected to a computer (Columbus Instruments, Columbus, Ohio, USA). The Lesion and Sham groups were pretreated with either 0.8 or $1.6 \mathrm{~g} / \mathrm{kg}$ IP of GLY or SAL $30 \mathrm{~min}$. before the behavioral testing. The locomotor activity of each animal was assessed during two testing periods as follows: (1) a novel environment (NOVEL), and (2) a MAP injection (MAP-INJ). For

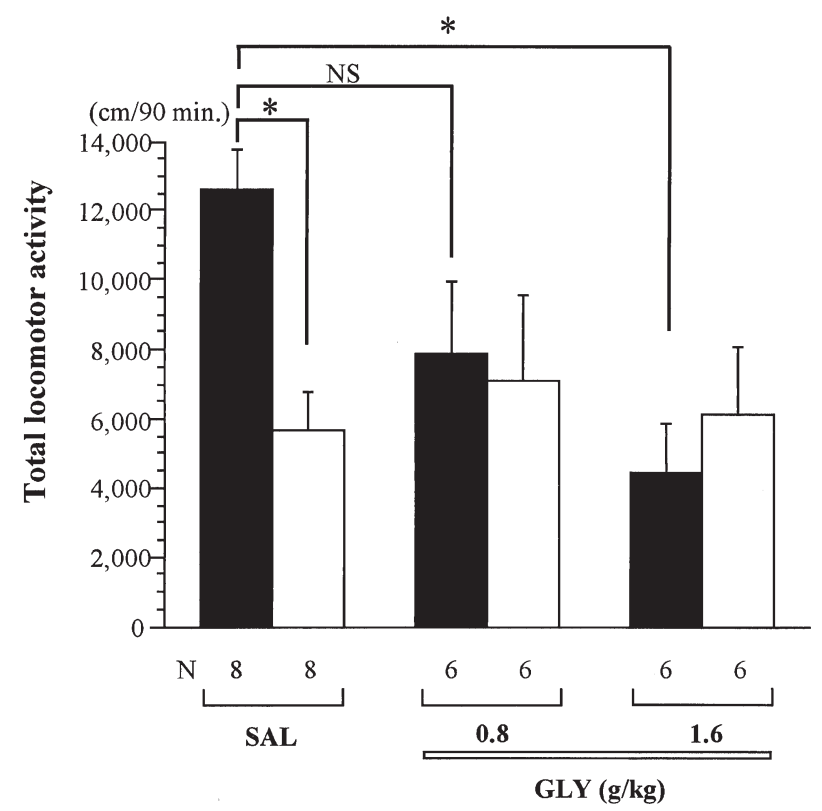

Figure 2. Methamphetamine-injection (MAP-INJ). After NOVEL, rats were injected with MAP $(1.5 \mathrm{mg} / \mathrm{kg}$, IP $)$, and locomotor activity of Sham (open-bar) or Lesion (closed-bar) rats was measured over the next 90 min (means \pm SEM). Newman-Keuls post-hoc comparisons were used for comparisons between groups $\left({ }^{*} p<.05\right)$. $\mathrm{N}$, the number of animals; NS, no significance.

NOVEL, rats were placed in an activity box for $60 \mathrm{~min}$ while the locomotor activity was measured. After NOVEL, rats were injected with MAP $(1.5 \mathrm{mg} / \mathrm{kg} \mathrm{IP})$, and locomotor activity was measured over the next 90 min. After lesion verification using cresyl violet staining, we deleted unsuccessfully lesioned rats from the statistical analysis (Kato et al. 2000). For statistical analysis, total distance traveled was analyzed by three-way analysis of variance (ANOVA) with STATUS (Lesion or Sham) and GLY (SAL, 0.8 or $1.6 \mathrm{~g} / \mathrm{kg}$ ) as independent variables and TREATMENT (NOVEL, MAP-INJ) as repeated measures. Newman-Keuls post-hoc comparisons were used for comparisons between groups. Differences at $p<.05$ were considered significant.

The findings from the overall ANOVA revealed a significant main effect for GLY $[\mathrm{F}(2,34)=4.73, p<.05]$ and TREATMENT $[\mathrm{F}(1,34)=93.83, p<.001]$. Although there were no significant main effects for STATUS $[\mathrm{F}(1,34)=4.05, p=.052]$, there was a significant twoway, GLY by STATUS interaction $[\mathrm{F}(2,34)=3.40, p<$ .05]. In addition, there were no other significant twoway or three-way interactions.

For the group tested with NOVEL alone, SAL-pretreated Lesion (SAL-Lesion) rats were hyperactive compared with SAL-Sham rats (Figure $1, p<.001$ ). GLY $(0.8$ and $1.6 \mathrm{~g} / \mathrm{kg}$ )-Lesion rats significantly reduced locomotor activity compared with SAL-Lesion rats (Fig- 
ure $1, p<.001)$. For the group tested with MAP-INJ alone, SAL-Lesion rats were hyperactive compared with SAL-Sham rats (Figure 2, $p<.05)$. GLY (1.6 but not $0.8 \mathrm{~g} / \mathrm{kg}$ )-Lesion rats showed significantly reduced locomotor activity compared with SAL-Lesion rats (Figure $2, p<.05$ ).

The novel finding of the present study was that GLY reduced NOVEL- and MAP-induced hyperlocomotion (corresponding to positive symptoms of schizophrenia) in $\mathrm{nVH}$ damaged rats. In a preliminary experiment, none of the tested doses of GLY $(0.8$ and $1.6 \mathrm{~g} / \mathrm{kg})$ could affect NOVEL-induced locomotor activity in intact rats (data not shown). Similarly, Javitt et al. (1997) reported that GLY $(0.8$ and $1.6 \mathrm{~g} / \mathrm{kg})$ could not affect amphetamine-induced hyperactivity in intact rats. Considering these findings, as well as the present observations, it is suggested that there are differences between intact rats and $\mathrm{nVH}$ lesioned rats (an animal model of schizophrenia) in the effects of GLY. Although we previously reported that this animal model showed hyperresponsiveness to PCP (Kato et al. 2000), there have been few studies on the dysfunction of NMDA-type glutamatergic neurotransmission. The present findings suggest that this animal model may be accompanied by the dysfunction of the GLY-sensitive sites of the NMDA receptor. It has been reported that strychnine-sensitive GLY sites will also affect dopaminergic neurotransmission in the rat brain (Yadid et al. 1993). Further studies are needed to clarify the interaction between NMDA and other neurotransmissions in this animal model.

Heresco-Levy et al. (1999) reported that GLY therapy led to a significant reduction in negative symptoms in patients with schizophrenia without exacerbating effects on positive or extrapyramidal symptoms. In contrast to the present findings, previous studies reported that GLY had no reducing effect on positive symptoms (Goff and Evins 1998; Heresco-Levy et al. 1999). The discrepancy in activity (or positive symptoms)-reducing effects of GLY between the present findings and theirs may be because all patients in those studies were receiving antipsychotic medications and that the maximal therapeutic effect on positive symptoms had already been achieved prior to GLY treatment. Therefore, the present findings raise the possibility that GLY therapy could improve positive symptoms in patients with schizophrenia.

In conclusion, GLY attenuated nVH-induced hyperactivity, and this effect was evident both in the presence and absence of MAP. The $\mathrm{nVH}$ lesions may result in a form of hyperactivity that differs from normal locomotion in the degree to which it is highly sensitive to regulation by GLY. Furthermore, these findings support the hypoglutamatergic hypothesis of schizophrenia, and suggest a novel approach for the pharmacotherapy of positive and negative symptoms in patients with schizophrenia.

\section{REFERENCES}

Goff DC, Evins AE (1998): Negative symptoms in schizophrenia: neurobiological models and treatment response. Harv Rev Psychiatry 6:59-77

Heresco-Levy U, Javitt DC, Ermilov M, Mordel C, Silipo G, Lichtenstein M (1999): Efficacy of high-dose glycine in the treatment of enduring negative symptoms of schizophrenia. Arch Gen Psychiatry 56:29-36

Javitt DC, Zukin SR (1991): Recent advances in the phencyclidine model of schizophrenia. Am J Psychiatry 148: 1301-1308

Javitt DC, Sershen H, Hashim A, Lajtha A (1997): Reversal of phencyclidine-induced hyperactivity by glycine and the glycine uptake inhibitor glycyldodecylamide. Neuropsychopharmacology 17:202-204

Johnson JW, Ascher P (1987): Glycine potentiates the NMDA response in cultured mouse brain neurons. Nature 325:529-531

Kato K, Shishido T, Ono M, Shishido K, Kobayashi M, Suzuki H, Nabeshima T, Furukawa H, Niwa SI (2000): Effects of phencyclidine on behavior and extracellular levels of dopamine and its metabolites in neonatal ventral hippocampal damaged rats. Psychopharmacology 150:163-169

Kleckner NW, Dingledine R (1988): Requirement for glycine in activation of NMDA-receptors expressed in Xenopus oocytes. Science 241:835-837

Lipska BK, Jaskiw GE, Weinberger DR (1993): Postpubertal emergence of hyperresponsiveness to stress and to amphetamine after neonatal excitotoxic hippocampal damage: A potential animal model of schizophrenia. Neuropsychopharmacology 9:67-75

Lipska BK, Weinberger DR (1996): Hippocampal damage in the neonatal rat as a model of some aspects of schizophrenia. In Kato N (eds), The Hippocampus: Functions and Clinical Relevance. Amsterdam, Elsevier, pp 465475

Yadid G, Pacak K, Golomb E, Harvey-White JD, Lieverman DM, Kopin IJ, Goldstein DS (1993): Glycine stimulates striatal dopamine release in conscious rats. Brit J Pharmacol 110:50-53 